\title{
Syphilitic Balanitis of Follmann: Can Dermoscopy Be Useful Tool in the Differential Diagnosis?
}

\author{
Roberta Vezzoni ${ }^{1}$, Claudia Colli ${ }^{2}$, Giacomo Rebez $^{3}$, Carlo Trombetta $^{3}$, Andrea Boltar $^{3}$, \\ Iris Zalaudek ${ }^{1}$, Claudio Conforti ${ }^{1}$
}

1 Dermatology Clinic, University of Trieste, Ospedale Maggiore di Trieste, Italy

2 MST Centre, ASUGI, Trieste, Italy

3 Department of Urology, University of Trieste, ASUGI, Trieste, Italy

Key words: syphilitic balanitis of Follmann, dermoscopy, dermatology, urology, sexually transmitted diseases

Citation: Vezzoni R, Colli C, Conforti C, et al. Syphilitic balanitis of Follmann: can dermoscopy be useful tool in the differential diagnosis? Dermatol Pract Concept. 2022;12(1):e2022022. DOI: https://doi.org/10.5826/dpc.1201a22

Accepted: June 2, 2021; Published: January 2022

Copyright: $\odot 2022$ Vezzoni et al. This is an open-access article distributed under the terms of the Creative Commons Attribution License BY-NC-4.0, which permits unrestricted noncommercial use, distribution, and reproduction in any medium, provided the original authors and source are credited.

Funding: None.

Competing interests: None.

Authorship: All authors have contributed significantly to this publication.

Corresponding author: Claudio Conforti, MD, Dermatology Clinic, Maggiore Hospital of Trieste, Trieste, Italy.

E-mail: Claudioconforti@yahoo.com

\section{Introduction}

Syphilis is a sexually transmitted infection caused by the spirochete Treponema pallidum that has different clinical presentations. Among them, there is Follmann balanitis, that was first described by Eugene Follmann in 1948. It is a rare manifestation of primary syphilis that presents as erosive balanitis [1]. Herein we report the dermoscopic description of a Follmann balanitis and discuss how to differentiate it from other sexually transmitted or genital diseases.

\section{Case Presentation}

A previously healthy 28 -year-old man was admitted to the Urology Department for the presence of painless erosions on the glans and erythema. He was initially treated with topic antibiotics and steroids but without benefit. Physical examination revealed painless crusted erosions of the glans and foreskin associated with edema and ulceration of the coronal sulcus (Figure 1A). Dermoscopy revealed the presence of homogeneously distributed glomerular vessels and focused linear curved vessels on an erythematous background with hyperpigmented post-inflammatory areas (Figure 1B). Bilateral inguinal lymphadenopathy was also observed. The patient reported a high-risk sexual contact 2 months earlier. A polymerase chain reaction test performed for common sexually transmitted diseases was negative. Serological tests for syphilis were positive: VDRL 1:32 and TPHA 1:1280. Based on these results, a diagnosis of Follmann syphilitic balanitis was made. The first choice of treatment for primary syphilis is a single intramuscular injection of 2.4 million units of benzathine-penicillin, but due to allergy reported by the patient, treatment with doxycycline $100 \mathrm{mg}$ twice daily for 14 days was started. At the one-month follow-up visit, complete healing of the glans was observed. 


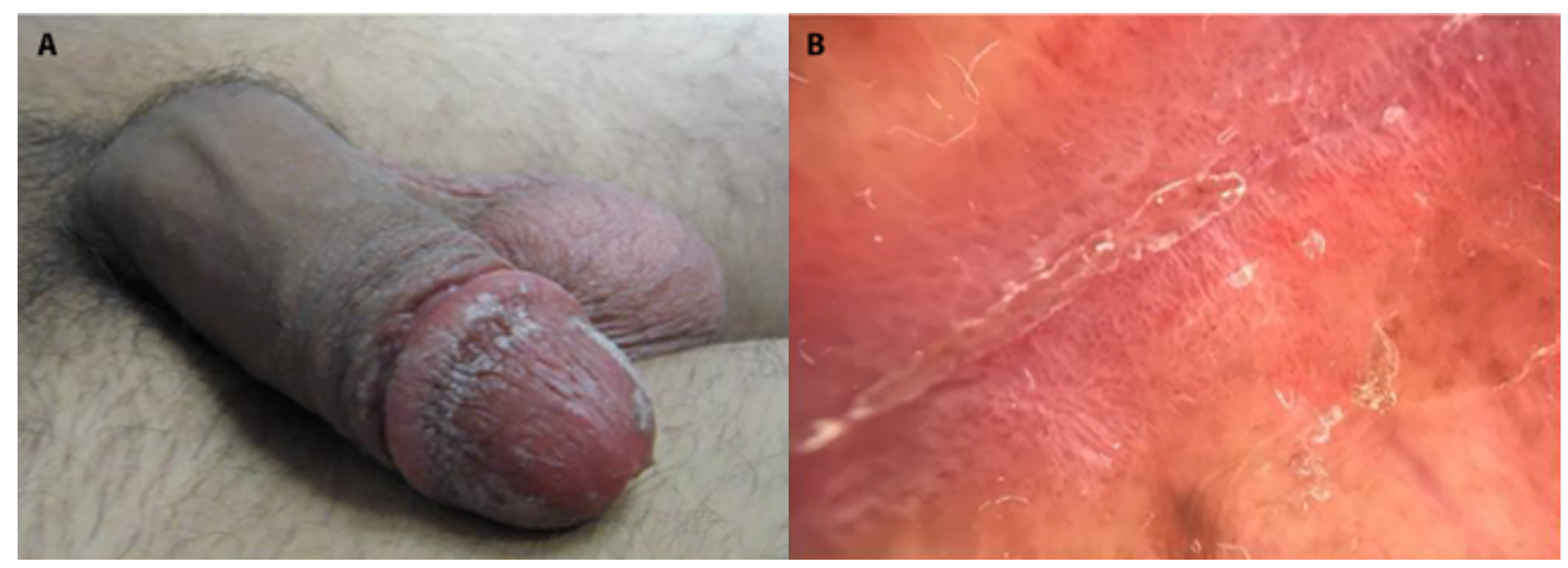

Figure 1. (A) Detail of the penis, glans, and foreskin. Importantly, note the erosive erythema with adherent crusts and the presence of a hardened ulceration compatible with a chancre in the coronal sulcus. (B) Dermoscopic evaluation of the coronal sulcus showing the presence of homogeneously distributed glomerular vessels and focused linear curved vessels on an erythematous background with hyperpigmented post-inflammatory areas.

\section{Conclusions}

The clinical features of syphilitic balanitis or balanoposthitis are variable, as they can manifest as an edematous balanitis with erosions and crusted lesions or as a papular form with smooth white/pink coalescent papules and plaques on the surface of the glans. Common clinical manifestations are the hardening of the glans penis and bilateral inguinal lymphadenopathy [1].

The differential diagnosis requires the exclusion of infectious such as Candida albicans, groups B and D Streptococci and herpes simplex virus, and noninfectious diseases such as lichen sclerosus et atrophicus, Zoon balanitis, psoriasis, eczema, fixed drug rash and erythroplasia of Queyrat (EQ). Even if dermoscopy is not the gold standard test for the diagnosis, it is a useful tool in the differential diagnosis. Candidal balanitis is characterized by cottage cheeselike structures and blurry linear vessels, psoriasis is defined by a quite monomorphic pattern with diffusely distributed dotted vessels. On the other hand, glomerular vessels are a constant finding in EQ while focused linear curved vessels and orange structureless areas are dermoscopic findings of
Zoon balanitis [2]. In our case, the presence of glomerular and hairpin vessels on an erythematous background without other typical signs of other pathologies helped us to exclude the differential hypotheses reported above.

Follmann erosive balanitis may be the only clinical expression of primary syphilis, so it is essential to include this rare clinical manifestation in the differential diagnosis of balanitis and balanoposthitis [1]. Dermoscopy could be an extremely useful tool that can direct the experienced clinician towards the correct diagnosis and allow proper treatment.

Informed consent: Written informed consent for publication of clinical details and clinical images was obtained from the patient.

\section{References}

1. Follmann E. Le problème de la balanite syphilitique. Ann Dermatol Syph. 1948;8:470-483.

2. Conforti C, Giuffrida R, Di Meo N, et al. Benign dermatoses of the male genital areas: A review of the literature. Dermatol Ther. 2020;33(3):e13355. DOI: 10.1111/dth.13355. PMID: 32239734. 